\title{
Referenciação aos cuidados de saúde secundários em idade pediátrica
}

Maria Inês Santos, ${ }^{1}$ Inês Coelho, ${ }^{2}$ Frederico Rosário, ${ }^{3}$ Patrícia Machado, ${ }^{4}$ Lurdes Nery, ${ }^{5}$ João Ribeiro, ${ }^{6}$ António Lemos ${ }^{5}$

\section{RESUMO}

Objectivos: Estimar a taxa de referenciação à consulta externa hospitalar em idade pediátrica das Unidades de Saúde Familiar (USF) Grão Vasco e Viriato ao Hospital de São Teotónio (HST); caracterizar a referenciação nestas USF à consulta externa do HST; analisar a resposta hospitalar.

Tipo de estudo: Observacional, transversal e analítico.

Local: HST, USF Grão Vasco e Viriato.

População: Crianças e adolescentes (menos de 18 anos de idade), inscritas nas USF Grão Vasco e Viriato, que utilizaram as consultas de Saúde Infantil e Juvenil durante o ano de 2009.

Métodos: Foram analisadas todas as referenciações efectuadas durante o ano de 2009. Os dados foram colhidos por consulta dos processos hospitalares e das USF. Variáveis estudadas: sexo, idade, número de crianças referenciadas por médico, número de consultas por médico, anos de prática do médico, consulta pedida, motivo de referenciação, iniciativa da referenciação, forma de escrita das cartas, qualidade das cartas, tempo até primeira consulta, informação de retorno.

Resultados: Foram analisadas 135 referenciações, $63 \%$ do sexo masculino, média de idades 6,1 $\pm 4,2$ anos. Foi encontrada uma taxa de referenciação de $1,1 \%(0,9 ; 1,3)$. Não se encontrou correlação significativa entre os anos de prática clínica do médico de família e a taxa de referenciação.

As especialidades mais referenciadas foram Pediatria Geral (35,6\%) e Cirurgia Pediátrica (33,3\%). As cartas de referenciação apresentaram boa qualidade em $69,6 \%$ dos casos. Verificou-se uma associação significativa entre a qualidade das cartas e a especialidade referenciada, tendo-se encontrado uma associação significativa entre a Cirurgia Pediátrica e cartas de qualidade razoável/má.

A mediana do tempo de espera pela consulta foi de 92 dias $(83,6 ; 100,4)$. Encontrou-se informação de retorno em $10,5 \%$ dos casos.

Conclusões: A taxa de referenciação encontrada foi inferior à observada noutros artigos. Existem aspectos a melhorar na qualidade das cartas de referenciação e no tempo de resposta hospitalar às consultas.

Palavras-Chave: Referenciação e Consulta; Comunicação; Cuidados de Saúde Primários; Hospital; Pediatria; Qualidade dos Cuidados de Saúde.

\section{INTRODUÇÃO}

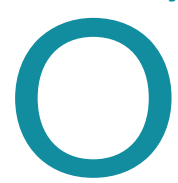

Médico de Família (MF) constitui um dos pilares fundamentais na organização estrutural e funcional dos serviços de saúde, constituindo a porta de entrada do utente/doente nos cuidados de saúde. ${ }^{1,2}$ Cabe ao MF fazer a avaliação inicial da situação clínica e decidir quanto à necessidade de referenciação aos cuidados secundários, tendo em conta as medidas já realizadas, os meios ao seu dispor e o âmbito da sua acção.

Dos diferentes objectivos dos dois níveis de cuidados nasce a complementaridade de acções. Numa al-

${ }^{4}$ Interna do $2 .{ }^{\circ}$ Ano do Internato Complementar de Medicina Geral e Familiar na USFViriato, Viseu;

${ }^{5}$ Assistente Graduado de Medicina Geral e Familiar na USF Grão Vasco, Viseu;

${ }^{6}$ Assistente de Medicina Geral e Familiar na USF Viriato, Viseu.

${ }^{2}$ Interna do 2. ${ }^{\circ}$ Ano do Internato Complementar de Medicina Geral e Familiar na USF 
tura em que cada vez mais se procura melhorar a qualidade do serviço prestado à comunidade e, ao mesmo tempo, racionalizar os escassos recursos humanos e tecnológicos, ${ }^{3}$ é importante que a interacção entre os diferentes intervenientes no serviço nacional de saúde seja eficaz. Dada a complexidade crescente dos vários problemas de saúde e o risco de utilização excessiva dos serviços, uma comunicação adequada entre os Cuidados de Saúde Secundários e os Cuidados de Saúde Primários (CSP) é considerada um bom indicador de qualidade de cuidados. ${ }^{3}$ Alguns autores verificaram a existência de falhas nesta articulação de cuidados no nosso país, salientando a importância de uma boa informação clínica, quer de referenciação, quer de retorno, com o objectivo da partilha da responsabilidade dos diversos profissionais na eficaz orientação do doente. ${ }^{2,3} \mathrm{Na}$ literatura não estão descritas taxas de referenciação para a idade pediátrica, ${ }^{2-5}$ nem se encontra analisada a comunicação entre os diferentes níveis de cuidados neste grupo etário particular.

Assim, este trabalho teve como objectivos: estimar a taxa de referenciação à consulta externa hospitalar em idade pediátrica das USF Grão Vasco e Viriato ao HST; caracterizar a referenciação em idade pediátrica nestas USF à consulta externa do HST; e analisar a resposta hospitalar às referenciações efectuadas.

\section{MÉTODOS}

Tipo de estudo: observacional, transversal, analítico.

Duração e período: a recolha de dados, relativos ao ano de 2009, foi efectuada entre um de Março e nove de Abril de 2010.

Local: estudo realizado no Hospital de São Teotónio, E.P.E. (HST), Unidade de Saúde Familiar (USF) Grão Vasco e USF Viriato.

População: crianças e adolescentes (menos de 18 anos de idade), inscritas nas USF Grão Vasco e Viriato, que utilizaram as consultas de Saúde Infantil e Juvenil durante o ano de 2009.

Amostra: de conveniência, constituída por todas as crianças e adolescentes referenciadas à consulta externa do HST pelas USF Grão Vasco e Viriato durante o ano de 2009.

Definição conceptual e operacional das variáveis

1.Sexo, operacionalizado em «Feminino» $\mathrm{e}$ «Masculino»;
2. Idade à data da referenciação, definida como número de anos completos à data da colheita dos dados exceptuando os lactentes ( $<1$ ano) nos quais a idade foi calculada de acordo com a equação

$$
\text { Idade }=\text { Número de meses completos } / 12
$$

Esta variável foi posteriormente operacionalizada em lactentes, 1-5 anos, 6-9 anos e adolescentes ( $>9$ anos). Optou-se por esta divisão por se tratar dos grupos etários utilizados no Serviço de Pediatria do HST;

3. Número de crianças referenciadas por médico, definida como o número total de referenciações efectuadas por cada MF nas USF Grão Vasco e Viriato durante o ano de 2009;

4. Número de consultas por médico, definida como o número total de consultas efectuadas por cada MF nas USF Grão Vasco e Viriato a crianças e adolescentes durante o ano de 2009;

5. Anos de prática do Médico de Família, definida como o número de anos completos de exercício da profissão Médico;

6. Consulta pedida, definida como a consulta solicitada na carta de referenciação, nas diferentes especialidades médicas encontradas nas cartas: Pediatria Geral, Cirurgia Pediátrica, Oftalmologia, Desenvolvimento, Pedopsiquiatria, Ortopedia, Otorrinolaringologia (ORL), Gastrenterologia e Dermatologia. Tendo em conta que muitas das especialidades referenciadas tinham poucos casos, optou-se por agrupar todas as que tinham 9 ou menos referenciações, de forma a poder estimar-se a mediana do tempo de resposta. Para este efeito assumiu-se que as consultas pedidas que aguardavam agendamento iriam coincidir com as consultas marcadas. Assim, a variável foi operacionalizada em: Pediatria Geral, Cirurgia Pediátrica, Oftalmologia e Outras;

7. Motivo de referenciação, definida como o motivo que levou ao pedido de consulta hospitalar, procedeuse à sua operacionalização em «Desenvolvimento» e «Somático» consoante o motivo de referência aos cuidados hospitalares fosse do foro do desenvolvimento/comportamento ou do foro físico, respectivamente;

8. Iniciativa da referenciação, definida como a entidade que sugeriu inicialmente a necessidade de refe- 
renciação, operacionalizada em «Médico de Família», «Família/Utente», «Escola/Infantário» e «Outras», avaliada através da consulta dos processos clínicos das USF;

9. Forma de escrita das cartas de referenciação. As cartas de referenciação foram enviadas através do sistema ALERT $\mathrm{P} 1^{\circledR}$ ou através de via postal, manuscritas ou dactilografadas. Esta variável foi definida de acordo com a forma como a carta foi elaborada: métodos informáticos ou manuscrita, operacionalizada em «Manuscrita» e «Dactilografada»;

10. Qualidade das cartas, avaliada através de consulta das cartas de referenciação integradas no processo clínico hospitalar, ${ }^{1,2}$ provenientes das USF Grão Vasco e Viriato, operacionalizada em «Boa», «Aceitável» e «Má» (Quadro I);

11. Tempo até primeira consulta, definida como o número de dias completos decorridos entre a data de envio da carta de referenciação e a data da primeira consulta hospitalar. Para aferir a data da primeira consulta foi consultado o sistema ALERT $^{\circledR}$ hospitalar;

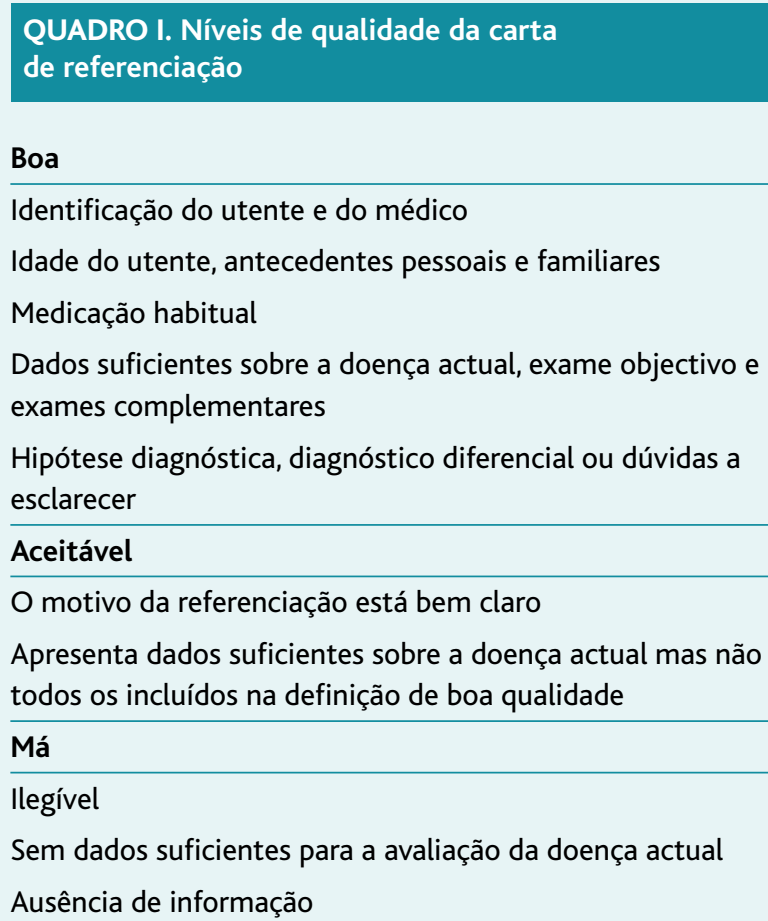

Adaptado de Arribas e colaboradores ${ }^{1}$ e Ponte e colaboradores ${ }^{2}$
12. Informação de retorno, definida como a presença da informação de retorno nos processos das USF das crianças e adolescentes que tinham recebido alta da consulta externa hospitalar, operacionalizada em «Presente» quando esta constava no processo e «Ausente» caso contrário.

Taxa de referenciação: Calculada pelo quociente entre o número de referenciações efectuadas a utentes com menos de 18 anos de idade e o número total de consultas efectuadas a utentes com menos de 18 anos de idade, ${ }^{2}$ durante $o$ ano de 2009, em ambas as USF. Estes dados foram obtidos através das aplicações informáticas Sonho ${ }^{\circledR}$ no HST, Vitacare ${ }^{\circledR}$ na USF Grão Vasco e $\mathrm{SAM}^{\circledR}$ na USF Viriato.

Recolha e tratamento dos dados: Todos os dados foram colhidos através de consulta dos processos hospitalares e dos das USF. Os dados obtidos foram codificados e registados no suporte informático Microsoft ${ }^{\circledR}$ Excel 2007 tendo depois sido tratados com os softwares estatísticos SPSS $15.0^{\circledast}$ (SPSS Inc, Chicago, IL) ${ }^{6} \mathrm{e} \mathrm{R}^{\circledR}$ 2.7.0. ${ }^{7}$

Análise dos dados: A descrição dos dados teve como base a distribuição de frequências, medidas de tendência central (médias e medianas) e medidas de dispersão (desvio-padrão). A comparação das variáveis qualitativas foi efectuada através do teste $\chi^{2}$ quando verificados os pressupostos para a sua utilização (dimensão amostral $>20$; todas as frequências esperadas $>1$; pelo menos $80 \%$ das frequências esperadas $\geq 5$ ), ou teste exacto de Fisher caso contrário. ${ }^{8}$ As variáveis contínuas foram comparadas com o teste $t$ de Student para 2 amostras independentes após verificação dos pressupostos de normalidade e homocedasticidade das amostras, tendo-se recorrido à correç̧ão de Welch no caso de violação deste último. ${ }^{8} \mathrm{~A}$ existência de correlação entre os anos de prática do médico e a taxa de referenciação foi estudada através do coeficiente de correlação Kendall's tau b. Optou-se pela utilização deste coeficiente de correlação pois os dados não apresentavam uma distribuição normal (invalidando a utilização do coeficiente $R$ de Pearson) e continham elevado número de empates (invalidando a utilização do coeficiente Ró de Spearman). ${ }^{9} \mathrm{~A}$ associação entre a qualidade das cartas de referenciação e as especialidades hospitalares, e entre a iniciativa da referenciação e o motivo de referenciação, foi avaliada através da análise de resíduos normalizados ajustados em ta- 
belas de contingência (considerou-se significativo se resíduo $<-1,96$ ou resíduo $>1,96$ ). A análise dos tempos de resposta hospitalar aos pedidos de consulta foi efectuada com recurso ao estimador Kaplan-Meier, tendo os tempos de resposta entre cada tipo de consulta sido comparados com o teste log-rank, com o valor $p$ corrigido pelo método de Hochberg quando se efectuaram comparações múltiplas. Para a tomada de decisão foi considerado o nível de significância $5 \%$.

\section{RESULTADOS}

\section{Caracterização da amostra}

Foram referenciadas 135 crianças, $63 \%$ do sexo masculino. As 43 crianças referenciadas pela USF Grão Vasco tinham idade média de 7,2 $\pm 5,0$ anos e as 92 referenciadas pela USF Viriato $5,7 \pm 3,7$ anos. A maioria das referenciações foi efectuada no grupo etário entre os 1 e 5 anos (38,5\%), seguido dos 6 aos 9 anos (31,9\%), adolescentes (20\%) e lactentes $(9,6 \%)$.

As USF estudadas revelaram-se homogéneas quanto à idade $\left(t_{\text {welch }}(64,7)=1,74 ; p=0,09\right)$ e sexo das crianças referenciadas $\left(\chi_{\text {Pearson }}^{2}(1)=2,43 ; p=0,12\right)$.

\section{Taxa de referenciação}

Foram efectuadas 12273 consultas no ano de 2009, $6287(51,2 \%)$ das quais na USF Viriato. A taxa de referenciação global foi de 1,1\% (IC95\%: [ 0,9; 1,3 ]), sendo de $1,46 \%$ na USF Viriato e de $0,72 \%$ na USF Grão Vasco. Foi encontrada uma diferença significativa entre as taxas de referenciação nas duas USF $\left(\chi_{\text {Pearson }}^{2}(1)=15,3 ; p\right.$ $<0,001)$. Os anos de prática clínica dos Médicos de Família das duas USF variaram entre 7 e 34 anos, não se tendo verificado relação entre a taxa de referenciação de cada MF e os respectivos anos de prática clínica (Kendall's tau $b=-0,095 ; p=0,62$ ).

\section{Características da referenciação}

As consultas mais solicitadas foram Pediatria Geral, Cirurgia Pediátrica e Oftalmologia. Na área médica, 41,5\% dos motivos de referenciação estavam relacionados com o desenvolvimento, como a perturbação de hiperactividade e défice de atenção, as dificuldades de aprendizagem, o atraso global do desenvolvimento, as dificuldades da linguagem e a dislexia. (Quadro II)

Da análise dos processos familiares nas USF apurou-se que a principal iniciativa da referenciação foi o
Médico de Família (77,8\%), seguida da Escola/Infantário $(12,6 \%)$, Família/Utente $(5,2 \%)$ e Outras $(4,4 \%)$.

Quando a referenciação não foi inicialmente proposta pelo MF, a patologia implicada foi maioritariamente ao foro do desenvolvimento e do comportamento (Quadro III). A análise das variáveis Iniciativa da referenciação e Motivo de referenciação, dicotomizada em Somático/Desenvolvimento, mostrou haver uma associação muito significativa entre elas (teste exacto de Fisher, $p<0,001)$. Pela análise dos resíduos em tabelas de contingência foi possível constatar que, quando os sintomas eram somáticos, a iniciativa da referenciação foi preferencialmente do $\mathrm{MF}$ (resíduo > 1,96), ao passo que, quando os sintomas eram do foro do desenvolvimento/comportamento, a iniciativa da referenciação teve como origem preferencial a Escola/Infantário e Outras fontes (resíduo > 1,96).

Analisando o conteúdo das cartas de referenciação, constatou-se que a maioria apresentava boa qualidade (69,6\%), com $15,6 \%$ dos casos de má qualidade. No Quadro IV podem verificar-se os dados que estavam presentes nas cartas de referenciação analisadas.

A maioria das cartas de má qualidade foram dirigidas à consulta de Cirurgia Pediátrica (Quadro V), tendo-se verificado uma relação significativa entre as cartas de referenciação apresentarem qualidade aceitável ou má e a referenciação à Consulta de Cirurgia Pediátrica $\left(\chi_{\text {Pearson }}^{2}(2)=20,3 ; p<0,001\right.$; resíduo $\left.>1,96\right)$.

A maioria das cartas de referenciação foi dactilografada $(77,3 \%)$, não se tendo verificado associação significativa entre a forma de escrita e a qualidade das cartas de referenciação $\left(\chi_{\text {Pearson }}^{2}(2)=1,96 ; p=0,38\right)$.

\section{Características da resposta hospitalar}

O tempo de espera até primeira consulta variou com a especialidade de referenciação, apresentando no global uma mediana de 92 dias, mínimo de 13 dias e máximo de 421 dias; $23 \%$ das referenciações aguardavam marcação à data de conclusão do estudo, variando os seus tempos de espera entre os 130 e os 449 dias. Uma vez que algumas especialidades tinham poucas referenciações, optou-se por agrupar as que tinham 9 ou menos referenciações, conforme descrito na secção «Métodos». Obteve-se assim uma nova distribuição das especialidades referenciadas: 52 referenciações a Pediatria Geral, 46 a Cirurgia Pediátrica, 14 a Oftalmolo- 


\begin{tabular}{|c|c|c|c|}
\hline Consulta Pedida & $\mathbf{n}$ & Motivo & $\mathbf{n}$ \\
\hline \multirow[t]{6}{*}{ Pediatria Geral } & \multirow[t]{6}{*}{$48(35,6 \%)$} & Dificuldades de aprendizagem & $8(5,9 \%)$ \\
\hline & & Má progressão ponderal & $7(5.2 \%)$ \\
\hline & & PHDA & $5(3,7 \%)$ \\
\hline & & Asma & $4(3 \%)$ \\
\hline & & Obesidade & $3(2,2 \%)$ \\
\hline & & Outros & $21(15,6 \%)$ \\
\hline \multirow[t]{6}{*}{ Cirurgia Pediátrica } & \multirow[t]{6}{*}{$45(33,3 \%)$} & Fimose & $14(10,4 \%)$ \\
\hline & & Anquiloglossia & $6(4,4 \%)$ \\
\hline & & Testículos não descidos & $5(3,7 \%)$ \\
\hline & & Hérnia Inguinal & $4(3 \%)$ \\
\hline & & Hemangioma & $3(2,2 \%)$ \\
\hline & & Outros & $13(9,6 \%)$ \\
\hline \multirow[t]{3}{*}{ Oftalmologia } & \multirow[t]{3}{*}{$14(10,4 \%)$} & Diminuição da acuidade visual & $8(5,9 \%)$ \\
\hline & & Estrabismo & $2(1,5 \%)$ \\
\hline & & Outros & $4(3 \%)$ \\
\hline \multirow[t]{3}{*}{ Desenvolvimento } & \multirow[t]{3}{*}{$7(5,2 \%)$} & PHDA & $5(3,7 \%)$ \\
\hline & & Atraso global do desenvolvimento & $1(0,7 \%)$ \\
\hline & & Dificuldades da linguagem & $1(0,7 \%)$ \\
\hline \multirow[t]{4}{*}{ Pedopsiquiatria } & \multirow[t]{4}{*}{$7(5,2 \%)$} & PHDA & $4(3 \%)$ \\
\hline & & Dislexia & $1(0,7 \%)$ \\
\hline & & Atraso global do desenvolvimento & $1(0,7 \%)$ \\
\hline & & Alterações do comportamento & $1(0,7 \%)$ \\
\hline \multirow[t]{2}{*}{ Ortopedia } & \multirow[t]{2}{*}{$5(3,7 \%)$} & Escoliose & $3(2,2 \%)$ \\
\hline & & Deformidades dos dedos & $2(1,5 \%)$ \\
\hline \multirow[t]{2}{*}{ ORL } & \multirow[t]{2}{*}{$5(3,7 \%)$} & Hipertrofia adenoideia & $3(2,2 \%)$ \\
\hline & & Hipoacúsia & $2(1,5 \%)$ \\
\hline \multirow[t]{2}{*}{ Gastrenterologia } & \multirow[t]{2}{*}{$3(2,2 \%)$} & Diarreia crónica & $2(1,5 \%)$ \\
\hline & & Hemorróidas & $1(0,7 \%)$ \\
\hline Dermatologia & $1(0,7 \%)$ & Molusco contagioso & $1(0,7 \%)$ \\
\hline
\end{tabular}

PHDA: perturbação de hiperactividade e défice de atenção

sultas de Pediatria, $42,9 \%$ de Oftalmologia, 71,7\% de Cirurgia Pediátrica e $73,9 \%$ de Outras especialidades.

Trinta dias após a referenciação haviam sido realizadas $4,4 \%$ das consultas, ascendendo a $21,5 \%$ aos 60 dias. Ao fim de 150 dias estavam por realizar $28,1 \%$ das consultas solicitadas. A análise por especialidades revelou que, ao fim deste tempo, Pediatria Geral tinha por realizar $1,9 \%$ das consultas solicitadas, $7,1 \%$ de Oftalmologia, $63 \%$ de Cirurgia Pediátrica e 30,4\% de Outras especialidades.

As medianas dos tempos de resposta hospitalar podem ser consultadas na Quadro VI.

Não foi possível calcular o Intervalo de Confiança $95 \%$ para a mediana da Consulta de Cirurgia Pediátrica, uma vez que $54,3 \%$ das consultas solicitadas a esta especialidade ainda aguardavam agendamento à data da conclusão do estudo (Figura 1).

A análise da Figura 1 mostra que as curvas dos tempos de resposta hospitalar das diferentes especialidades parecem ser semelhantes até cerca dos 70 dias, altura em que começam a divergir dois grupos: o primeiro, composto pelas especialidades de Oftalmologia e Pediatria, com os menores tempos de resposta hospitalar; o segundo, constituído pela especialidade de Cirurgia Pediátrica e pelo grupo das restantes especialidades, correspondendo a tempos de resposta mais elevados. Este último grupo parece começar a divergir após os 130 dias, apresentando a Cirurgia Pediátrica

gia e 23 para Outras Especialidades. Nenhuma consulta foi marcada durante os primeiros oito dias. Doze semanas após a data da referenciação aguardavam consulta 56,3\% das referenciações. Analisando a resposta hospitalar por especialidade, verificou-se que ao fim de doze semanas estavam por realizar $38,5 \%$ das contempos de resposta mais elevados. A aplicação do teste log-rank ao conjunto dos tempos de resposta mostrou haver pelo menos um tipo de consulta que diferia dos restantes $(p<0,001)$ pelo que se procedeu a comparações múltiplas entre os diferentes tempos de marcação, tendo o valor $p$ sido corrigido pelo método de 


\begin{tabular}{|c|c|c|}
\hline \multicolumn{3}{|c|}{$\begin{array}{l}\text { QUADRO III. Motivos de Consulta nos casos em que a iniciativa de } \\
\text { referenciação não foi o Médico de Família }\end{array}$} \\
\hline Motivo & Iniciativa da Referenciação & $\mathbf{n}$ \\
\hline \multirow[t]{2}{*}{ PDHA } & Escola/Infantário & $9(6,7 \%)$ \\
\hline & Outros & $2(1,5 \%)$ \\
\hline Dificuldades da linguagem & Escola/Infantário & $3(2,2 \%)$ \\
\hline Atraso global do desenvolvimento & Escola/Infantário & $3(2,2 \%)$ \\
\hline \multirow[t]{2}{*}{ Dislexia } & Escola/Infantário & $2(1,5 \%)$ \\
\hline & Outros & $1(0,7 \%)$ \\
\hline Alterações do comportamento & Pais/Utente & $1(0,7 \%)$ \\
\hline \multirow[t]{2}{*}{ Sinais/Sintomas do pénis } & Pais/Utente & $1(0,7 \%)$ \\
\hline & Outros & $1(0,7 \%)$ \\
\hline Deformidade osteo-articular & Pais/Utente & $3(2,2 \%)$ \\
\hline Tumefacção labial & Pais/Utente & $1(0,7 \%)$ \\
\hline Unha Encravada & Pais/Utente & $1(0,7 \%)$ \\
\hline Estrabismo & Outros & $2(1,5 \%)$ \\
\hline
\end{tabular}

PHDA: perturbação de hiperactividade e défice de atenção

\section{QUADRO IV. Informação presente nas cartas de} referenciação

\begin{tabular}{l|c} 
Elementos da carta de referenciação & $\mathbf{n}$ \\
\hline Identificação do Utente & $135(100 \%)$ \\
\hline Identificação do médico & $135(100 \%)$ \\
\hline Idade do Utente & $134(99,3 \%)$ \\
\hline História da Doença Actual & $110(81,5 \%)$ \\
\hline Exame Objectivo & $104(77 \%)$ \\
\hline Dúvidas a esclarecer & $97(71,9 \%)$ \\
\hline Antecedentes Pessoais & $80(59,3 \%)$ \\
\hline Exames Auxiliares de Diagnóstico & $41(30,4 \%)$ \\
\hline Antecedentes Familiares & $28(20,7 \%)$ \\
\hline Diagnóstico provável & $28(20,7 \%)$ \\
\hline Medicação & $18(13,3 \%)$ \\
\hline Diagnóstico diferencial & $6(4,4 \%)$
\end{tabular}

Hochberg. Assim, foram encontradas diferenças significativas no tempo de resposta entre os diferentes tipos de consulta excepto entre as de Pediatria Geral e Oftalmologia, e entre as de Cirurgia Pediátrica e Outras Con- sultas (Quadro VII), não se confirmando a existência de diferença significativa entre estas duas últimas.

Das referenciações efectuadas, $23 \%$ aguardavam marcação. A maioria destas $(80,6 \%)$ era dirigida à consulta de Cirurgia Pediátrica.

Até à data da conclusão do estudo tinham tido alta 38 crianças, tendo-se encontrado informação de retorno em 10,5\% dos casos (IC95\%: [ 3,4\%; 23,5\% ]).

\section{DISCUSSÃO}

\section{Taxa de referenciação}

A taxa de referenciação encontrada neste estudo foi inferior às estimadas em estudos anteriores, que variam entre os $5,1 \%$ e os $10,1 \% .^{2-5}$ Contudo, estes estudos abordam a taxa de referenciação global e não apenas a pediátrica. A diferença encontrada está provavelmente relacionada com o menor número de patologias encontradas nas crianças quando comparadas com os adultos, em particular com os mais idosos. Um achado inesperado deste estudo relaciona-se com a diferença encontrada nas taxas de referenciação entre as duas USF. Apesar da sua pro-

QUADRO V. Qualidade das cartas de referenciação de
acordo com especialidade referenciada
\begin{tabular}{l|r|r|r}
\hline \multicolumn{3}{c}{ Qualidade } \\
\hline Especialidade & \multicolumn{1}{|c}{ Boa } & \multicolumn{1}{c}{$\begin{array}{c}\text { Aceitável } \\
\text { n }\end{array}$} & \multicolumn{1}{c}{ Má } \\
\hline Pediatria Geral & $44(32,6 \%)$ & $2(1,5 \%)$ & $2(1,5 \%)$ \\
\hline Cirurgia Pediátrica & $20(14,8 \%)$ & $12(8,8 \%)$ & $13(9,6 \%)$ \\
\hline Desenvolvimento & $7(5,2 \%)$ & 0 & 0 \\
\hline Pedopsiquiatria & $4(3 \%)$ & 0 & $3(2,2 \%)$ \\
\hline Ortopedia & $4(3 \%)$ & 0 & $1(0,7 \%)$ \\
\hline Otorrinolaringologia & $1(0,7 \%)$ & $4(3 \%)$ & 0 \\
\hline Oftalmologia & $11(8,1 \%)$ & $2(1,5 \%)$ & $1(0,7 \%)$ \\
\hline Gastrenterologia & $2(1,5 \%)$ & 0 & $1(0,7 \%)$ \\
\hline Dermatologia & $1(0,7 \%)$ & 0 & 0 \\
\hline Total & $94(69,6 \%)$ & $20(14,8 \%)$ & $21(15,6 \%)$ \\
\end{tabular}




\begin{tabular}{|c|c|c|c|}
\hline \multicolumn{4}{|c|}{$\begin{array}{l}\text { QUADRO VI. Medianas dos tempos de resposta } \\
\text { hospitalar às consultas solicitadas e respectivos } \\
\text { intervalos de confiança }\end{array}$} \\
\hline Consultas & Mediana & Erro-Padrão & IC95\% \\
\hline Global & 92 & 4,3 & $83,6-100,4$ \\
\hline Pediatria Geral & 77 & 2,8 & $71,5-82,5$ \\
\hline Oftalmologia & 78 & 1,9 & $74,3-81,7$ \\
\hline Outras & 131 & 4,8 & $121,6-140,4$ \\
\hline Cirurgia Pediátrica & 304 & $a$ & d \\
\hline
\end{tabular}

adados insuficientes para o cálculo do Intervalo de Confiança 95\% IC95\%: Intervalo de Confiança 95\% para a mediana dos tempos de resposta hospitalar

ximidade geográfica e da homogeneidade encontrada nas idades e sexo das crianças referenciadas, a taxa de referenciação estimada para a USFViriato foi o dobro da USF Grão Vasco. Poder-se-ia argumentar que esta diferença estaria relacionada com os anos de prática clínica dos Médicos de Família. No entanto, não foi encontrada relação significativa entre a taxa de referenciação e os anos de prática clínica. Uma possível explicação para esta discrepância poderá estar relacionada com as datas de inicio de funções das respectivas USF. A USF Grão Vasco iniciou funções a 23 de Outubro de 2006 e a USF Viriato a 9 de Dezembro de 2008. Após estas datas, as respectivas USF continuaram a incluir nas suas listas novos utentes sem MF, possivelmente com patologias já estabelecidas mas ainda por diagnosticar. Tendo em conta que os dados foram colhidos relativamente ao ano de 2009, é concebível que a USF Viriato tenha apresentado uma maior taxa de referenciação. Ou seja, para além da incidência habitual de problemas de saúde, em teoria semelhante nas duas USF, os médicos da USF Viriato poderão ter sido confrontados com uma maior prevalência de problemas de saúde não diagnosticados. Este fenómeno não terá assim sido observado na USF Grão Vasco, pois muitos destes problemas terão sido diagnosticados durante os anos de 2007 e 2008. Esta hipótese, não considerada durante a fase de planeamento do estudo, alerta para a necessidade de ter em conta o tempo de formação das listas de utentes do MF em estudos de referenciação, podendo em parte ser responsável pela discrepância encontrada nas taxas de referenciação estimadas, quer neste estudo, quer noutros já publicados. Seria ainda importante que estudos futuros investigassem outros motivos para estas diferenças, avaliando o efeito de outras variáveis socio-demográficas das listas utentes e dos MF nas taxas de referenciação.

\section{Características da referenciação}

À semelhança do que foi verificado em estudos anteriores, ${ }^{1-5}$ a maioria das referenciações foi dirigida a

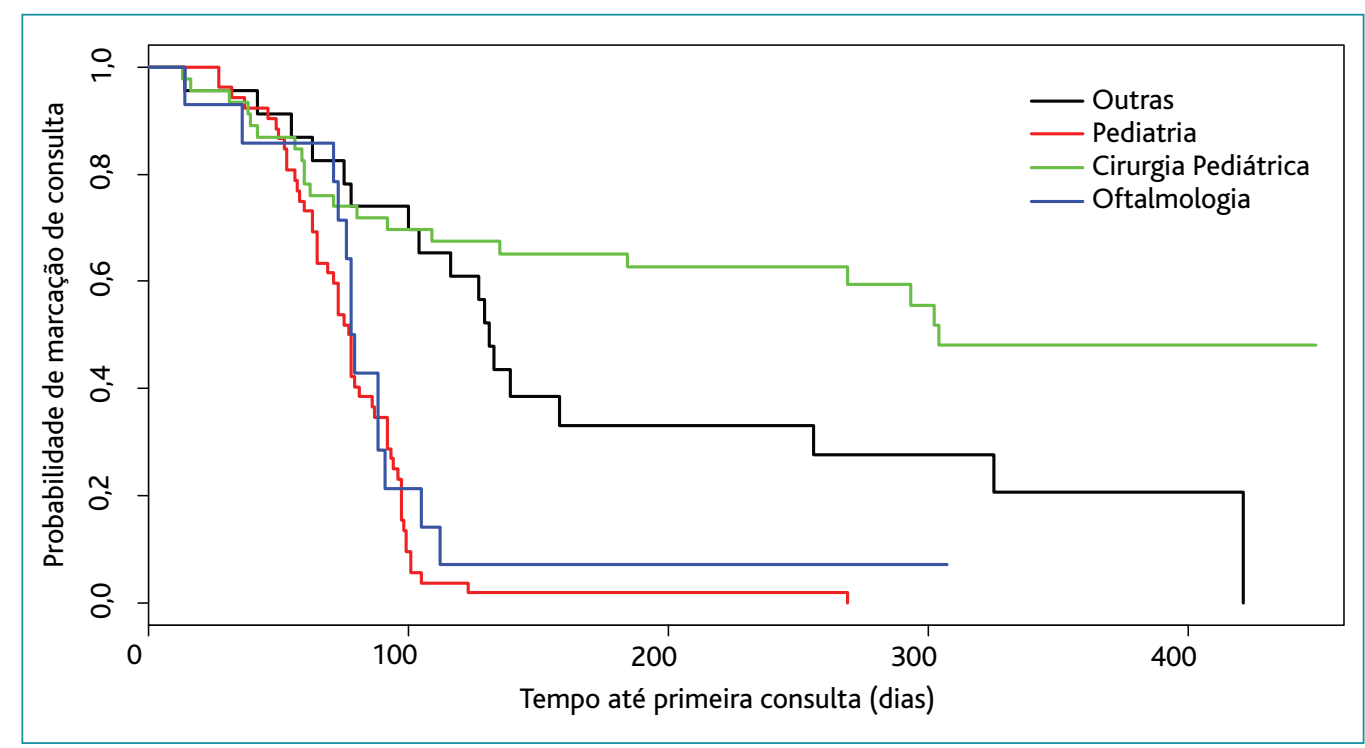

Figura 1. Estimativas de Kaplan-Meier da proporção de referenciações sem consulta marcada para cada especialidade. 
especialidades cirúrgicas ou médico-cirúrgicas, onde são utilizadas técnicas específicas de diagnóstico e/ou tratamento não disponíveis nos CSP.

$\mathrm{Na}$ área médica, uma parte significativa das referenciações deveu-se a problemas do foro do desenvolvimento. Os resultados obtidos mostraram que este tipo de problemas é

QUADRO VII. Comparação entre os tempos de resposta das Consultas
\begin{tabular}{l|r|r|r|r|} 
Consultas & Outras & Pediatria Geral & Cirurgia Pediátrica & Oftalmologia \\
\hline Outras & - & & & \\
\hline Pediatria Geral & $26,1(<0,001)$ & - & & \\
\hline Cirurgia Pediátrica & $4,6(0,064)$ & $40,0(<0,001)$ & - & \\
\hline Oftalmologia & $7,5(0,019)$ & $1,0(0,312)$ & $11,6(0,003)$ & - \\
\hline
\end{tabular}

Nota: o primeiro valor em cada célula corresponde à estatística do teste log-rank e o segundo ao respectivo valor $p$ corrigido pelo método de Hochberg. essencialmente identificado a nível da escola/infantário, ao passo que o MF identifica maioritariamente os problemas de índole somática. Isto pode ser explicado: pelo maior contacto dos professores/educadores com a criança quando comparado com o tempo que o MF passa com a criança em consulta, devido ao grande intervalo temporal entre as consultas preconizadas pela Direcção Geral da Saúde; pelo facto das crianças recorrerem aos CSP, entre as consultas definidas pela Direcção Geral de Saúde, essencialmente por problemas somáticos; e por os professores/educadores possuírem hoje formação específica para a detecção deste tipo de problemas, o que os leva a identificá-los antes mesmo dos próprios pais. Uma solução possível para aumentar a detecção precoce deste problema seria a integração, em todas as unidades de CSP, de consultas de Psicologia em idades-chave para avaliação formal do desenvolvimento e comportamento. Actualmente, o Agrupamento de Centros de Saúde Dão Lafões I integra nos seus quadros profissionais apenas uma psicóloga, insuficiente para a implementação deste tipo de programa.

A análise da qualidade das cartas de referenciação revelou que $69,6 \%$ delas apresentava boa qualidade, bastante superior à encontrada em estudos anteriores, em que a percentagem de cartas de boa qualidade variou entre $9,4 \%$ e $20,6 \% .^{1,2}$ Poder-se-ia pensar que a melhoria da qualidade das cartas fosse o resultado da implementação da referenciação por via informática, que automaticamente assume alguns dos critérios de avaliação de qualidade, como por exemplo a legibilidade ou a identificação do utente, ao passo que os estudos anteriores avaliaram essencialmente cartas manuscritas. Contudo, não encontrámos diferenças significativas na qualidade entre as cartas de referenciação manuscritas e enviadas por via informática, o que atesta a favor de uma evolução na qualidade da referenciação. Estas melhorias devem ser analisadas com alguma cautela uma vez que os estudos anteriores abrangem todas as referenciações e não apenas as efectuadas em idade pediátrica.

Uma análise mais detalhada das cartas de referenciação permitiu verificar que a qualidade destas não se distribuiu de forma homogénea pelas diferentes especialidades, pois foi encontrada uma associação significativa entre as cartas de pior qualidade e a referenciação à consulta de Cirurgia Pediátrica. Esta associação ficou a dever-se ao facto de na maioria destas cartas constarem apenas a identificação, idade e diagnóstico provável. Estes resultados mostram a necessidade de alertar os profissionais de saúde para este problema, com o intuito de melhorar a informação presente nas cartas de referenciação, e assim melhorar o serviço prestado ao utente. Num estudo anterior, em que foram detectadas lacunas na qualidade da informação das cartas de referenciação, ficou demonstrado que a realização de reuniões de serviço de avaliação e discussão das cartas de referenciação melhorou significativamente a qualidade das mesmas. ${ }^{1}$ Este poderia ser um tema a incluir periodicamente nas reuniões das USF visando a melhoria contínua da informação presente nas cartas. Estudos futuros deverão procurar identificar as eventuais barreiras à melhoria da qualidade das cartas, bem como desenhar estratégias que as permitam ultrapassar.

\section{Características da resposta hospitalar}

A mediana global do tempo de espera encontrada 
neste estudo foi 92 dias. É difícil fazer comparações com outros estudos, uma vez que estes utilizam principalmente médias, abrangem todos os grupos etários, apresentam critérios de inclusão e exclusão heterogéneos, e não têm em conta as consultas que aguardam marcação para o seu cálculo. A bibliografia consultada apresenta tempos médios de espera que variam entre 29 e 82,5 dias. ${ }^{3,10}$

Alguns estudos referem como indicador de qualidade a resposta aos oito dias e às doze semanas. ${ }^{3,10}$ Neste estudo nenhuma criança tinha sido observada ao fim de oito dias, valor semelhante ao encontrado num estudo anterior, ${ }^{3}$ no qual $94 \%$ das consultas ainda não tinham sido realizadas ao fim deste tempo. Por outro lado, ao fim de doze semanas, verificámos que 56,3\% ainda não tinham tido uma primeira consulta. Outros estudos encontraram também elevadas percentagens de consultas por realizar ao fim deste tempo, variando entre $36,6 \%$ e $58 \%{ }^{3,10,11}$

A Portaria n. ${ }^{\circ}$ 1529/2008, de 26 de Dezembro, ${ }^{12}$ define os tempos máximos de resposta garantidos para todo o tipo de prestações de cuidados de saúde sem carácter de urgência. Para os hospitais do SNS, o tempo é dividido, consoante o nível de prioridade atribuído pela triagem hospitalar, em 30 dias (consulta de realização muito prioritária), 60 dias (prioritária) e 150 dias (normal). Não tendo tido acesso à prioridade atribuída a cada referenciação pudemos apenas verificar que cerca de $28 \%$ das consultas não tinham respeitado os 150 dias admitidos para uma referenciação de prioridade normal fixados pela portaria citada. No entanto, devese também ter em conta que cerca de um quinto das consultas foi realizada num prazo máximo de 60 dias, pelo que se admite a possibilidade de as referenciações consideradas prioritárias ou muito prioritárias estarem a ter resposta dentro dos tempos estipulados. Estudos futuros que abordem a resposta hospitalar às referenciações dos CSP poderão clarificar estes aspectos devendo para o efeito ter acesso às prioridades atribuídas a cada referenciação.

A análise dos tempos de resposta hospitalar por especialidades veio mostrar a existência de dois grupos distintos: um primeiro constituído por Pediatria e Oftalmologia, com cerca de $60 \%$ das consultas realizadas ao fim de doze semanas e mais de $90 \%$ das consultas realizadas antes dos 150 dias; um segundo grupo, en- globando a Cirurgia Pediátrica e o conjunto das restantes especialidades, com cerca de $30 \%$ das consultas realizadas ao fim de doze semanas e com uma importante percentagem de consultas com tempo de resposta acima dos 150 dias ainda por efectuar. $\mathrm{O}$ facto de a Cirurgia Pediátrica ter um tempo mediano de espera duas vezes superior ao tempo máximo de espera definido por portaria para prioridade normal deve ter em conta que no HST existe apenas um profissional com a especialidade de Cirurgia Pediátrica. Não foi possível calcular o intervalo de confiança para a mediana do tempo de espera para esta especialidade uma vez que cerca de $50 \%$ dos casos aguardavam ainda agendamento à data da conclusão do estudo.

Importa também referir que no HST a triagem é feita por médicos e portanto é concebível que os tempos de resposta, que variaram entre um mínimo de 13 dias e um máximo de 421 dias, poderão estar relacionados com a informação clínica disponível na carta de referenciação e não apenas com a capacidade de resposta hospitalar.

Não foi possível determinar a mediana do tempo de espera para cada uma das especialidades englobadas no grupo Outras especialidades por apresentarem poucas referenciações. No entanto, a sua análise conjunta sugere que o tempo de espera está acima da mediana geral. Estudos futuros devem ter em conta esta limitação e alargar o tempo de estudo ou o número de USF/Centros de Saúde estudados.

Neste estudo foi encontrada uma taxa de informação de retorno de $10,5 \%$. Outros estudos publicados encontraram taxas de retorno mais elevadas, entre os $26,3 \%$ e os $36,6 \% .{ }^{2,3,10}$ Estudos futuros devem ter em conta este aspecto no sentido de identificar as suas causas para futura correcção. A inexistência de informação de retorno limita a prestação de cuidados prestados pelo MF, podendo levar a tratamentos inadequados, prestação de informação contraditória ao doente, encaminhamento inadequado para o serviço de urgência e solicitação de novas consultas, o que resulta numa inadequada gestão dos recursos. A informação de retorno deve constituir um veículo de continuidade assistencial sendo, no entanto, muitas vezes descurada pelo médico hospitalar, devido à pressão assistencial a que está sujeito, o que o poderá levar a descuidar o seu dever e a considerar este documento como um mero passo burocrático. ${ }^{13}$ Deve-se ter em atenção que, no caso parti- 
cular da Pediatria, muita informação é veiculada através do boletim de saúde infantil e juvenil e não foi nosso objectivo avaliar este meio de comunicação, pelo que a taxa de informação de retorno obtida poderá estar subestimada. É de salientar que no HST, desde Janeiro de 2010, todas as altas na Consulta de Pediatria Geral têm nota de alta enviada ao MF por correio com história clínica, evolução, diagnóstico, exames efectuados e indicações importantes para seguimento.

\section{Pontos fortes e limitações do estudo}

Pode ser apontado como ponto forte deste estudo a metodologia estatística usada para estimar o tempo de resposta hospitalar às solicitações por parte dos CSP. Os estudos anteriormente publicados optaram por estimar este tempo através de médias. Esta metodologia é menos precisa por dois motivos: em primeiro lugar, o tempo até determinado evento não assume geralmente uma distribuição normal, sendo muito influenciada por valores extremos; em segundo, as referenciações que aguardam agendamento não podem ser contabilizadas, perdendo-se informação preciosa. O uso deste tipo de análise permite que estudos futuros usando a mesma metodologia possam comparar de forma mais fidedigna os seus resultados. Pode ainda ser referida como mais-valia deste estudo o facto de estudar em particular as referenciações em idade pediátrica, levando a uma análise mais precisa dos problemas específicos da referenciação neste grupo etário.

Devem ser consideradas algumas limitações deste estudo. Em primeiro lugar trata-se de uma amostra de conveniência referente apenas ao ano de 2009 das duas USF estudadas. Este facto limita a extrapolação dos resultados para outras unidades prestadores de CSP. Por outro lado, para estimar o tempo de resposta assumiuse que as consultas que aguardavam agendamento iriam ser coincidentes com consulta marcada. Admitimos que esta metodologia possa ter conduzido a algum viés na estimativa. Contudo, a maioria das referências que ainda não tinham consulta agendada eram de Cirurgia Pediátrica (cerca de $80 \%$ ) e não foram encontradas diferenças entre as referências a Cirurgia Pediátrica e a consulta efectivamente marcada. Como tal, para efeitos da estimação da mediana do tempo de resposta hospitalar o viés, a existir, terá sido mínimo. Devese considerar também como limitação o facto da «ini- ciativa da referenciação» ter sido verificada através da consulta do processo clínico da USF. É possível que os resultados possam não corresponder à realidade, uma vez que o MF poderá não ter registado a iniciativa da referenciação quando esta não tenha sido sua. É também possível que a estimativa da taxa de resposta hospitalar encontrada tenha sido influenciada por factores não investigados como perda da informação pelo doente, ter sido incorrectamente arquivada noutro processo ou pelo facto de não terem sido analisados os BSIJ. Por fim, o sistema de classificação aplicado para avaliar a qualidade das cartas, apesar de já ter sido usado em estudos anteriores, está sempre sujeito a algum grau de subjectividade.

Em conclusão, podemos dizer que a taxa de referenciação encontrada foi inferior à observada noutros artigos, e que existem aspectos passíveis de ser melhorados, por um lado no que respeita à qualidade das cartas de referenciação, por outro no tempo de resposta hospitalar às consultas solicitadas.

\section{REFERÊNCIAS BIBLIOGRÁFICAS}

1. Rubio Arribas $V$, Rodríguez Ibáñez ML, Sampedro Martínez E, Victores Benavente C, Alechiguerra García A, Barrio Gamarra JL. Evaluación de la calidad de comunicación eentre niveles asistenciales mediante el documento interconsulta. Aten Primaria. 2000 Dec; 26 (10): 681-4.

2. Ponte CM, Moura BG, Cerejo AC, Braga R, Marques I, Teixeira A, et al. Referenciação aos cuidados de saúde secundários. Rev Port Clin Geral 2006 Set-Out; 22 (5): 555-68.

3. Janeiro M. Acesso aos cuidados de saúde secundários numa extensão do Centro de Saúde de Serpa: 10 anos depois. Rev Port Clin Geral 2001 Mai-Jun; 17 (3): 193-207.

4. Forrest $C B$, Nutting PA, Starfield B, von Schrader S. Family physicians' referral decisions: results from the ASPN referral study. J Fam Pract 2002 Mar; 51 (3): 215-22.

5. de Prado Prieto L, García Olmos L., Rodriguez Salvanés F, Otero Puime A. Evaluación de la demanda derivada en atención primaria. Aten Primaria 2005 Feb 28; 35 (3): 146-51.

6. SPSS 15.0 for Windows Release 15.0.0 (6 Sep 2006).

7. R Development Core Team (2009). R:A language and environment for statistical computing. R Foundation for Statistical Computing, Vienna, Austria. ISBN 3-900051-07-0. Disponível em: http://www.R-project.org [acedido em 21/09/2011].

8. Marôco J. Análise Estatística com o PASW Statistics. Pêro Pinheiro: Joãao Marôco Livros; 2010.

9. Pestana M, Gageiro J. Análise de Dados para Ciências Sociais: a complementaridade do SPSS. $5^{\text {a }}$ ed. Lisboa: Sílabo; 2008.

10. Barreiro S. Referenciação e comunicação entre cuidados primários e secundários. Rev Port Clin Geral. 2005 Nov-Dez; 21 (6): 545-53.

11. Sá A, Jordão J. Estudo europeu sobre referenciação em cuidados pri- 
mários III - comparações internacionais. Rev Port Clin Geral 1994; 11: 115-24.

12. Portaria n 1529/2008, de 26 de Dezembro. "Diário da República, $1^{\text {a }}$ série - $\mathrm{N}^{\circ} 249 "$. p. 9040-4.

13. Buxadé Marti I, Canals Innamorati J, Montero Alcaraz JC, Pérez Galindo J, Bolíbar Ribas I. El informe de alta hospitalaria en atención primaria (I): análisis de su utilidad. Aten Primaria 2000 Oct; 26 (6): 383-8.

\section{CONFLITOS DE INTERESSES}

O autor Frederico Rosário é revisor convidado da Revista Portuguesa de
Clínica Geral e declara não ter estado envolvido no processo de decisão editorial para este artigo.

\author{
ENDEREÇO PARA CORRESPONDÊNCIA \\ Maria Inês Santos \\ Av. Rei D. Duarte 3504-509 Viseu \\ E-mail: mines.santos82@gmail.com
}

\section{ABSTRACT}

\section{AN ANALYSIS OF REFERRALS FROM PRIMARY CARE TO A PEDIATRIC HOSPITAL OUTPATIENT CLINIC}

Objectives: To determine the rate of referral of pediatric patients from the Grão Vasco Family Health Unit and the Viriato Family Health Units (FHU) to the São Teotónio Hospital (STH) pediatric outpatient clinic, to characterize these referrals, and to analyze the hospital's response to these referral.

Design: Cross-sectional

Setting: Two family health units and one pediatric hospital outpatient clinic.

Population: Children and adolescents under 18 years of age registered at the Grão Vasco and Viriato Family Health Units who attended the child and adolescent outpatient clinic at São Teotónio Hospital in 2009.

Methods: We reviewed all pediatric referrals made during 2009. Data were collected by examining hospital and FHU clinical records. The variables assessed were: gender, age, number of children referred per physician, number of consultations per physician, physician's years of practice, consult requested, reason for referral, initiator of referral, format of the referral letter, quality of referral letter, time to first consultation, and hospital feedback.

Results: A total of 135 referrals were analyzed. $63 \%$ of patients referred were male. The mean age of referred patients was 6.1 \pm 4.2 years. A referral rate of $1.1 \%(0.9 ; 1.3)$ was found. No significant correlation between years of clinical practice of the family physician and the rate of referral was found.

The specialties with the greatest number of referrals were general pediatrics (35.6\%) and pediatric surgery (33.3\%). The referral letters were of good quality in $69.6 \%$ of cases. There was a significant association between the quality of letters and the referred specialty. There was a significant association between referrals to pediatric surgery and letters with reasonable or bad quality. The median waiting time for consultation was 92 days $(83.6 ; 100.4)$. A reply to the referral from the hospital to the referring physician was found in $10.5 \%$ of cases.

Conclusions: The referral rate in this study was lower than that found in other studies. The quality of referral letters and the hospital's response time require improvement.

Keywords: Referral and Consultation; Communication; Primary Health Care; Hospital; Pediatrics; Quality of Health Care. 